\title{
Postmenopausal women with irritable bowel syndrome (IBS) have more severe symptoms than premenopausal women with IBS
}

\author{
Adrienne Lenhart ${ }^{1}$, Bruce Naliboff ${ }^{1,2}$, Wendy Shih $^{3}$, Arpana Gupta ${ }^{1,2}$, Kirsten Tillisch ${ }^{1,2}$, \\ Cathy Liu ${ }^{1,2}$, Emeran A. Mayer ${ }^{1,2}$, Lin Chang ${ }^{1,2}$ \\ ${ }^{1}$ Vatche and Tamar Manoukian Division of Digestive Diseases, David Geffen School of Medicine, \\ University of California, Los Angeles, CA, USA \\ ${ }^{2} \mathrm{G}$ Oppenheimer Center for Neurobiology of Stress and Resilience, University of California, Los \\ Angeles, CA, USA \\ ${ }^{3}$ Semel Institute for Neuroscience and Human Behavior, University of California, Los Angeles, \\ CA, USA
}

\begin{abstract}
Background-Although irritable bowel syndrome (IBS) is more common in women, little is known about the role of hormonal changes and menopause in IBS. This study aimed to evaluate for differences in gastrointestinal (GI) and psychological symptoms between pre- and postmenopausal women with IBS compared to age-matched men with IBS.

Methods-Patients with Rome-positive IBS were identified. Premenopausal women were <45 years of age with regular menses. Postmenopausal women were $\geq 45$ years without menses for at least 1 year. Younger men were $<45$ years, and older men were $\geq 45$ years. Questionnaires measured severity of IBS symptoms, somatic symptoms, health-related quality of life (HRQOL), and psychological symptoms. Multivariable linear or logistic regressions evaluating relationships between age and sex were performed.
\end{abstract}

Key Results-190 premenopausal women (mean age 30.25 years), 52 postmenopausal women (mean age 54.38 years), 190 men $<45$ years (mean age 30.45 years), and 52 men $\geq 45$ years (mean age 53.37 years) were included. Postmenopausal IBS women had greater severity of IBS symptoms $(P=.003)$ and worse physical HRQOL $(P=.048)$ compared to premenopausal women.

Correspondence: Lin Chang, G Oppenheimer Center for Neurobiology of Stress and Resilience, Vatche and Tamar Manoukian Division of Digestive Diseases, 10833 Le Conte Avenue, CHS 42-210, Los Angeles, CA 90095-7378, USA.

LinChang@mednet.ucla.edu.

AUTHOR CONTRIBUTIONS

$\mathrm{AL}$ and LC designed the study, wrote and edited the manuscript, and interpreted the data. BN assisted in the study design, interpreted the data, involved in statistical analysis, and edited and revised the manuscript. WS involved in statistical analysis. AG edited the manuscript. KT interpreted the data and edited the manuscript. CL contributed to research participant coordination and organization of data. EAM interpreted the data and edited the manuscript.

CONFLICT OF INTEREST

The authors have no personal or financial conflicts of interest to declare.

SUPPORTING INFORMATION

Additional supporting information may be found online in the Supporting Information section. 
No differences were observed between age-matched older and younger IBS men. Constipation increased with age for both sexes but was the principal IBS subtype in women only.

Conclusions and Inferences-Postmenopausal women with IBS have more severe IBS symptoms than premenopausal women, while no comparable age-related changes were seen in IBS men. The modulatory effect of female sex hormones on brain-gut interactions which affect visceral perception and GI function likely contributes to these findings.

\section{Graphical Abstract}

Postmenopausal Women with IBS Have More Severe IBS Symptoms

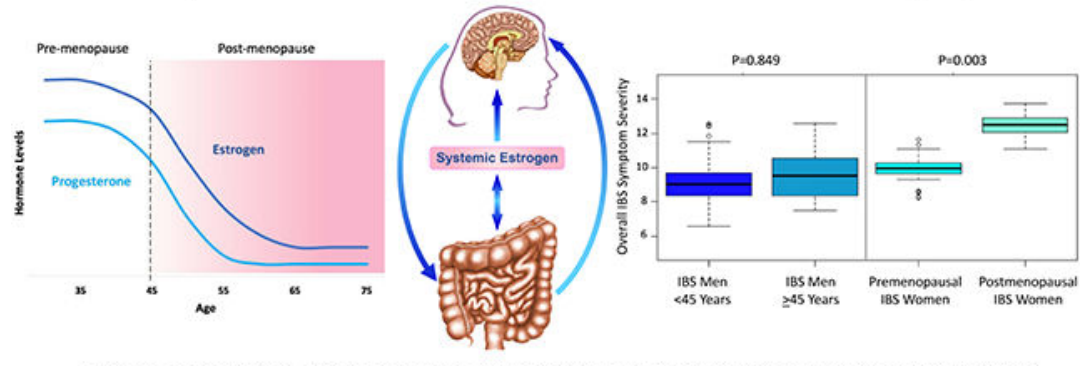

Post-menopausal Decline in Sex Hormones May Modulate the Brain-Gut Axis and Increase Severity of IBS

\section{Keywords}

estrogens; irritable bowel syndrome; menopause; menstrual cycle; progesterone; sex characteristics

\section{1 | INTRODUCTION}

Irritable bowel syndrome (IBS) is a chronic gastrointestinal (GI) condition characterized by recurrent abdominal pain and changes in stool form or frequency and is now considered to be a disorder of brain-gut interactions. ${ }^{1}$ This syndrome represents one of the most commonly diagnosed medical conditions in both primary care and gastroenterology clinics, with a prevalence reaching around $11 \% .^{2}$ Not only do women in the United States and other Western countries seek more health-care related services for IBS symptoms than do men, ${ }^{3}$ but many clinical and population-based studies have also demonstrated that the prevalence of IBS is higher in women compared to men. ${ }^{4}$ Women with IBS are more likely to experience increased GI symptoms of abdominal distention, bloating, nausea, and constipation, ${ }^{5-9}$ and women have also been described as having more severe extraintestinal symptoms, more frequent psychological symptoms of depression and anxiety, and lower health-related quality of life (HRQOL) than men with IBS. ${ }^{6,7,10-13}$ On the other hand, men with IBS typically report more episodes of diarrhea than do women with IBS. The explanations for these sex differences in IBS expression are likely multifactorial and include sex hormone-related influences and sex-related differences in brain-gut interactions that affect the stress response, visceral perception, and GI motility, among other factors. ${ }^{10}$

Gastrointestinal symptoms in premenopausal women with and without IBS may vary with phases of the menstrual cycle. ${ }^{14-17}$ Based on limited data, menstruating women with IBS appear to have more severe GI symptoms, looser stools, ${ }^{18}$ and increased visceral 
hypersensitivity ${ }^{17}$ during the late luteal and early menses phases, which are periods characterized by low or declining levels of estrogen and progesterone. Women with IBS also frequently report a coexistence of other menstrual cycle-associated conditions, including dysmenorrhea and premenstrual distress syndrome. ${ }^{15}$ Despite these observations, the physiologic role that estrogen and progesterone exert upon the GI tract, the brain-gut axis, and in the modulation of pain perception is not well understood. However, there are several proposed mechanisms through which sex hormones may affect the experience of pain, not only in IBS patients, but also in patients with fibromyalgia, ${ }^{19}$ migraines,${ }^{20}$ and other forms of chronic pain. ${ }^{21-23}$ Estradiol has been shown to have both pronociceptive and antinociceptive effects ${ }^{24}$ on pain perception, through its modulation of neurotransmitter systems and ascending arousal signals, enhancement of corticolimbic inhibitory pathways, stimulation of endogenous opioid systems, and its influence on intrinsic primary afferent nerves and extrinsic spinal receptors. ${ }^{24-27}$ Fluctuating levels of estradiol and progesterone may in fact be partially responsible for the dynamic changes in IBS and migraine-related symptoms ${ }^{20}$ during the menstrual cycle. In addition to pain modulation, both animal and human studies have also suggested that sex hormones may slow gastric emptying ${ }^{28-32}$ and intestinal transit, ${ }^{28,30,33}$ alter mucosal blood flow, ${ }^{34}$ and modulate visceral sensitivity. ${ }^{35}$ However, other studies have not confirmed such relationships. ${ }^{36-38}$

Menopause provides a physiologic state for studying the effects of declining levels of estradiol and progesterone on IBS symptoms. Despite the well-established physiologic changes associated with menopause, little is known about the impact of the menopause transition on GI symptoms and IBS. Evidence from the non-IBS population suggests that postmenopausal women may experience substantial differences in bowel function, as well as increased laxative use, gas, and heartburn compared to premenopausal women. ${ }^{39}$ However, there have only been a few studies directly comparing GI symptoms in pre- and postmenopausal women with IBS, and overall, these studies have demonstrated mixed results. ${ }^{12,40,41}$ In addition, a few of these studies were performed with relatively small sample sizes and did not directly compare symptom differences in younger and older men with IBS. While the transition to menopause is highly variable, there is some evidence to suggest that women with IBS in menopause or perimenopause experience more severe abdominal distention, bloating, and gas than do premenopausal women. ${ }^{40}$

Overall, studies evaluating the role of hormonal changes and the menopause transition in IBS are lacking. The primary aim of this study was to evaluate for differences in overall and individual IBS symptoms between premenopausal and postmenopausal IBS women in comparison with age-matched IBS men. By also comparing symptom differences in younger and older IBS men, we aimed to assess age-related differences in GI symptoms unrelated to the effects of estrogen and progesterone. Additional secondary aims were to (a) assess for differences in somatic and psychological symptoms and mental and physical HRQOL between pre- and postmenopausal women with IBS in comparison with men with IBS and (b) determine whether GI and non-GI symptoms varied according to menstrual cycle phase in premenopausal women. We hypothesized that there would be increased overall IBS symptom severity, abdominal pain, bloating, psychological, and somatic symptoms in postmenopausal women compared to premenopausal women but not in age-matched older 
vs younger men with IBS. Furthermore, we hypothesized that there would be an age-related increase in constipation in both men and women with IBS.

\section{2 | MATERIALS AND METHODS}

\section{1| Participants}

IBS patients between the ages of 18 and 69 years in our research database were consecutively screened for possible inclusion in this study. The majority of patients in the research database were recruited through community advertisements for physiologic or treatment-related clinical studies by the UCLA G. Oppenheimer Center for Neurobiology of Stress and Resilience, while a lesser proportion of patients were recruited for research studies directly through the general GI clinics at UCLA. Patient data from previous physiologic studies were utilized if patients met the inclusion criteria for this study. All participants were recruited during the time periods between September 1996 and November 2018.

All subjects underwent a medical history and physical examination. The diagnosis of IBS was made using the current Rome criteria at the time of a subjects' examination $(19.6 \%$ Rome II, 78.5\% Rome III, 1.9\% Rome IV). ${ }^{42-44}$ The diagnosis of IBS was further determined by exclusion of organic disease and through confirmation by a clinician with expertise in IBS. Participants with IBS were further classified as having constipationpredominant IBS (IBS-C), diarrhea-predominant IBS (IBS-D), IBS with mixed symptoms (IBS-M), or IBS unclassified (IBS-U) based on Rome subclassification criteria. IBS patients were comprised of premenopausal women with IBS, postmenopausal women with IBS, and men with IBS who were matched for menopausal age. Menopausal status was determined based on self-reported responses to a health-history questionnaire. Premenopausal women were defined as those patients under the age of 45 years who reported regular monthly menses for at least 1 year. Postmenopausal women were greater than or equal to the age of 45 years and could not have experienced menses for at least 1 year. Men with IBS were matched by menopausal age to the women with IBS, with the younger age group being men under 45 years and the age-matched older group being men greater than or equal to 45 years.

\section{2 | Menstrual cycle phase}

Menstrual cycle phase at the time of data collection was calculated using data collected on a woman's last menstrual period (LMP). Menses was defined as days 1-3 of the menstrual cycle, the follicular phase was defined as days $4-13$ of the menstrual cycle, and the luteal phase was defined as days 14 through completion of the menstrual cycle. Information regarding premenopausal women's use of oral contraceptive pills (OCPs) and postmenopausal women's use of HRT was also collected.

\subsection{Questionnaries}

2.3.1 Bowel Symptom Questionnarie-The Bowel Symptom Questionnaire (BSQ), which has been validated in IBS, ${ }^{45}$ was administered once to each patient at their research screening visit. The BSQ incorporated Rome II, III, or IV questions for IBS depending on the year of administration and was used to assess the severity of current overall IBS 
symptoms, to inquire about specific GI symptoms of abdominal pain and bloating, and to characterize IBS symptom-free periods. ${ }^{46,47}$ Current overall IBS symptoms were defined as those symptoms experienced during the previous week using a numerical rating scale from 0 to 20 , with 0 corresponding to "no symptoms" and 20 referring to the "most intense symptoms imaginable." Similar numeric rating scales were used to measure current abdominal pain and bloating. Duration of periods when IBS patients were relatively symptom-free over time included responses of $<1$ hour, up to 24 hours, between 1 and 3 days, between 4 and 7 days, up to 1 month, or up to 1 year. Bowel habits were further characterized by the percentage of time that patients had fewer than three bowel movements per week, more than three bowel movements a day, hard or lumpy stools, loose or watery stools, straining, urgency, or a sensation of incomplete evacuation.

2.3.2 Somatic Symptom Severity-Somatic symptoms were evaluated using the Patient Health Questionnaire (PHQ-15), a fifteen-item somatic symptom severity scale. ${ }^{48}$ The symptoms assessed included stomach pain, back pain, joint pain, menstrual cramps, headaches, chest pain, dizziness, fainting spells, feeling of a racing/pounding heart, shortness of breath, pain during sexual intercourse, constipation/loose bowels/diarrhea, nausea/gas/indigestion, fatigue, and trouble sleeping. Patients indicated how extensively they were bothered by each symptom, with 0 representing not bothered at all, 1 representing bothered a little, and 2 representing bothered a lot. Total scores on the PHQ-15 for somatic symptom severity ranged from 0 to 30 .

2.3.3 Health-Related Quality of Life (HRQL) - Health-related quality of life was assessed using the Short Form Health-Survey (SF-12) checklist, which assessed health status in the areas of general health, physical functioning, physical role, emotional role, and bodily pain. The domains selected from the SF-12 originate from a longer version of this questionnaire, the SF-36. ${ }^{49}$ Physical components of QOL were evaluated with the SF-12 Physical Composite Score (SF-12 PCS), and mental components of QOL were assessed with the SF-12 Mental Composite Score (SF-12 MCS). The raw scores for both the SF-12 PCS and SF-12 MCS were both converted to a 0- to 100-point scale (mean $=50$; standard deviation $=10$ ), with lower scales indicating poorer QOL. ${ }^{50}$

2.3.4 Psychological Symptoms-Current negative affect symptoms were assessed using the Hospital Anxiety and Depression Scale (HADS). Separate scores were calculated for HADS anxiety (ranging from 0 to 21 ) and depression (again ranging from 0 to 21 ). ${ }^{51}$

\subsection{Statistical analysis}

Clinical characteristics were summarized and stratified by groups (pre/postmenopausal and sex) status as count (\%) and mean (standard deviation [SD]). Categorical variables were compared with group status using a chi-squared test and continuous variables using a $t$-test or Wilcoxon test. Multivariable linear or logistic regressions were performed to determine the association between GI symptoms, somatic and psychological symptoms, and mental and physical HRQOL with premenopausal and postmenopausal IBS women in comparison with age-matched IBS men, adjusting for race, body mass index (BMI), and education. A $P$ value of $<.05$ was considered to be statistically significant. 


\section{3 | RESULTS}

\subsection{Patient characteristics}

Of the 691 IBS patients screened for inclusion in the study, there were a total of 376 premenopausal women, 52 postmenopausal women, 190 men under the age of 45 years, and 73 men at or over the age of 45 years. In order to more accurately compare pre- and postmenopausal women with men of similar age groups, we randomly matched patients by age and sex. A total of 190 premenopausal women with IBS, 52 postmenopausal women with IBS, 190 men with IBS under the age of 45 years, and 52 men with IBS at or over the age of 45 years were included in the final analysis. Patient characteristics are shown in Table 1. The mean age of premenopausal women was $30.25 \pm 6.99$ years, while the mean age for postmenopausal women was $54.38 \pm 5.3$ years. Among the men age-matched with the premenopausal women, the mean age was $30.45 \pm 7.32$ years, while the mean age for the men age-matched with the postmenopausal women was $53.37 \pm 5.22$ years. There were significant differences in race/ethnicity in the four groups of patients $(P<.001)$, including a larger percentage of Asian patients in the premenopausal women and younger men groups. However, the largest race category in each group was Caucasian (56.84\% of premenopausal women, $53.85 \%$ of postmenopausal women, $41.58 \%$ of the group of younger men, and $40.38 \%$ of the group of age-matched older men). There were no significant differences in the use of medications between the groups, including use of fiber, laxatives, antidiarrheal agents, antispasmodics, antidepressants, or benzodiazepines. There were also observed differences seen in BMI and educational status, but the variables of race, BMI, and education were controlled for during statistical analyses.

\section{2 | IBS symptoms}

There was no significant relationship between age and gender when evaluating IBS bowel habit predominance in younger and age-matched older men compared to pre- and postmenopausal women (Figure 1; $P>.05$ ). However, the majority of pre- and postmenopausal IBS women were classified as having IBS-C, and the percentage of women who reported predominantly constipation symptoms increased with menopausal age. While the majority of men in both the younger and older age-matched groups were classified as having IBS-D, the percentage of men also appeared to become more constipated with increasing age (Table 1, Figure 1). Although there was no significant change in bowel habit subtypes with age in men or women, postmenopausal women did report significantly less loose or watery stools than did premenopausal women $(P=.031)$ (Table S1).

Postmenopausal women with IBS had a greater severity of overall IBS symptoms compared to premenopausal women $(12.39 \pm 4.51$ vs $9.98 \pm 4.27 ; P=.003)$, whereas there was no difference in the severity of overall IBS symptoms in age-matched older vs younger men with IBS ( $9.48 \pm 4.33$ vs $9.51 \pm 4.57 ; P>.05)$ (Table 2, Figure 2$)$. We also compared individual abdominal symptoms and relative symptom-free periods between patient groups. There were no significant differences in abdominal pain and bloating scores between postmenopausal women compared to premenopausal women with IBS or between agematched older and younger men (Table 2). With regard to general periods when IBS patients 
were relatively symptom-free, there were no significant differences between pre-and postmenopausal women or between younger and older age-matched men (Table S2).

\subsection{Psychological and somatic symptoms}

Average depression and anxiety scores for our samples were well below the cutoffs for probable depression or anxiety disorders. However, premenopausal women with IBS did show lower scores on the depression scale (HADS depression) than postmenopausal women with IBS ( $3.27 \pm 2.87$ vs $4.2 \pm 3.28 ; P=.041)$, whereas depression scores were similar among younger and age-matched older men with IBS $(4.44 \pm 3.81$ vs $4.55 \pm 3.13 ; P=.316)$ (Table 2, Figure 3). Only 11 (5.79\%) premenopausal women, 3 (5.77\%) postmenopausal women, and $12(6.32 \%)$ younger men reported taking any type of antidepressant at the time of the study. No age-matched older men indicated that they were taking antidepressants. Only 4 (2.10\%) premenopausal women, $2(3.85 \%)$ postmenopausal women, and $9(4.74 \%)$ younger men were taking benzodiazepines at the time of the study, and no age-matched older men reported taking benzodiazepines. There was no significant difference in current anxiety scores (HADS anxiety) or somatic symptom severity (PHQ-15) in premenopausal women with IBS compared to postmenopausal women with IBS, nor in age-matched younger compared to age-matched older men (Table 2).

\subsection{Health-related quality of life}

Postmenopausal women with IBS expressed significantly lower physical HRQOL scores compared to premenopausal women with IBS (43.62 \pm 9.42 vs $49.04 \pm 9.18$, respectively; $P$ $=.048)$ (Table 2, Figure 4). However, there was no significant difference in physical HRQOL scores for age-matched older compared to younger men. In addition, there were no differences observed for mental HRQOL between pre- and postmenopausal women with IBS or between younger and age-matched older men with IBS (Table 2).

\section{5 | Menstrual cycle phase}

Out of the 190 premenopausal women with IBS included in this study, LMP data were available for $122(64.2 \%)$ patients. This was used to estimate the menstrual cycle phase at the time of the study. The clinical characteristics of this group did not differ from the ones who did not have LMP data. Of these IBS subjects, 12 patients were in menses, 74 patients were in the luteal phase, and 36 patients were in the follicular phase of the menstrual cycle. Of the 190 total premenopausal women, 42 (22.10\%) were taking OCPs. Of the 122 premenopausal women with LMP data, 32 patients (26.2\%) were on OCPs. The distribution of patients on OCPs was similar among the different phases of the menstrual cycle (4 patients in menses [33.3\%], 11 patients in the follicular phase [30.6\%], and 18 patients in the luteal phase (24.3\%), $P=.615)$.

Given that so few patients were actively in menses at the time of the study and the fact that previous studies have demonstrated that menses and premenses are associated with increased severity of GI symptoms, patients in active menses were excluded from the analysis of menstrual cycle phase. No significant differences between the luteal phase or follicular phase were discovered for age, current overall IBS symptom severity, abdominal pain, bloating, GI symptom-related anxiety, psychological symptoms, or HRQOL (Table 3). 


\section{4 | DISCUSSION}

While it is well established that women with IBS are more likely to experience increased GI symptoms compared to men, ${ }^{4}$ the effects that hormonal changes during the menstrual cycle and during menopause have on IBS symptoms have not been well studied. This study addressed these gaps in knowledge. Our study's main findings were that postmenopausal women with IBS had significantly greater severity of overall IBS symptoms, as well as worse physical HRQOL compared to premenopausal women. However, there was a lack of significant differences between age-matched older and younger men with IBS, so these findings do not appear to be age-related.

The reasons for increased GI symptoms after menopause may be multifactorial; however, our results are suggestive of a possible influence of low estrogen/progesterone levels or other female-specific effects on heightened postmenopausal GI symptom severity. While previous studies comparing pre- and postmenopausal women with IBS have consistently assessed for differences in specific GI symptoms such as abdominal pain, bloating, or intestinal gas, few have specifically evaluated overall IBS symptom severity. Only one prior study assessed this relationship and found no significant difference in the intensity of GI symptoms between pre- and postmenopausal women using the IBS-Severity Scoring System. ${ }^{41}$ However, this study did not directly assess symptom severity in pre- and postmenopausal women compared with age-matched men and the sample size only included 45 premenopausal and 16 postmenopausal women. Our study's larger sample size and comparison of IBS symptom severity between pre- and postmenopausal women and age-matched men may therefore provide stronger evidence for a possible declining sex hormone effect on IBS symptom severity.

Interestingly, menstrual cycle phase did not have any significant effect on IBS symptom differences within premenopausal women in our study. Previous studies in premenopausal women with IBS have suggested that more severe symptoms, looser stools, and increased visceral hypersensitivity may occur during the late luteal and early menses phases, periods that are characterized by low levels of estrogen and progesterone. ${ }^{14-17,52}$ However, because we did not observe any menstrual cycle variations in GI symptoms during the follicular and luteal phases, we can conclude that our findings of decreased IBS symptom severity and higher physical QOL in premenopausal women compared to postmenopausal women were unlikely to have been influenced by symptom fluctuations during the menstrual cycle.

Given that IBS is a disorder of brain-gut interactions, ${ }^{1}$ the modulatory role of estrogen on brain-gut pathways may result in both an increased predisposition of women to develop IBS and an enhanced visceral perception during low estrogen states (eg, menopause). Although the precise mechanism underlying the effect of estrogen and its metabolites on human brain networks are highly complex and incompletely understood, estrogen is known to modulate a number of neurotransmitter systems which have been implicated in IBS pathophysiology including acetylcholine, dopamine, norepinephrine (NE, produced in the locus coeruleus), and serotonin (5-HT, produced in the dorsal raphe nucleus). ${ }^{25}$ Estradiol has been shown to have both pronociceptive and antinociceptive effects on the psycho-physiologic response to pain. ${ }^{24}$ Estradiol mediates an increase in NE signaling to the brain which increases arousal, 
which may be a key mechanism in the predisposition to IBS ${ }^{53}$ in premenopausal women. However, estradiol has been shown to have a stimulatory effect on the endogenous opioid system function ${ }^{26}$ and is also thought to enhance corticolimbic inhibitory pathways (eg, reduce amygdala responsiveness and increase hippocampal activity) that attenuate emotional arousal and salience networks. ${ }^{24,26}$ It is conceivable that low estrogen states, such as menopause and premenses, are associated with a reduction both in ascending arousal signals and a simultaneous reduction in central inhibitory effects on emotional arousal networks, which outweighs the reduced effects of the ascending pathways from the brainstem. In addition, sex hormones may also exert effects on visceral pain sensitivity via $5-\mathrm{HT}_{3}$ receptors on intrinsic primary afferent nerves, extrinsic spinal receptors, and vagal primary afferent nerves. ${ }^{27}$ Taken together, these estrogen-dependent mechanisms could explain the increase in IBS symptoms and symptom severity during menopause.

The modulatory effects of estrogen on central arousal networks may also partially explain the poorer physical HRQOL found in postmenopausal IBS women compared to premenopausal women with no similar findings in the comparable IBS men groups. Extensive literature suggests a prominent role of the locus coeruleus and its central ascending noradrenergic projections in the pathophysiology of increased emotional arousal and stress responsiveness, ${ }^{54-56}$ physiological and psychological concepts that are important in defining QOL. However, there are likely multiple additional factors outside of declining hormone levels that contribute to poorer physical HRQOL scores after menopause, including deteriorating physical health, changing physical appearance, and increasing life adversities. While depression scores were significantly lower in premenopausal women compared to postmenopausal women, it should be noted that the average depression symptom scores in our study were overall well below the cutoff generally accepted for probable clinical depression, ${ }^{57}$ and the significance of our findings should therefore be interpreted within this context. Our results were unlikely to have been affected by medical therapy for depression, as only a minority of participants reported antidepressant use.

Individual IBS symptoms, including abdominal pain and bloating, were similar between preand postmenopausal women and younger and age-matched older men with IBS. These findings were similar to previous studies that also found no significant difference in abdominal pain between menstruating women and postmenopausal women with IBS. ${ }^{12,40,41}$ Some studies have reported more frequent episodes of abdominal distention or bloating in postmenopausal compared to premenopausal women with IBS, ${ }^{40,58}$ while others describe no difference. ${ }^{12,41}$ The lack of significance may have resulted from a relatively small sample size, and larger studies are therefore warranted.

Despite no significant differences in abdominal pain and bloating, we cannot rule out that sex hormone changes during the transition to menopause are contributing to differences in overall symptom severity. It should be noted that IBS symptom severity is a global measure that encompasses biopsychosocial factors, possible cultural or racial factors, ${ }^{59}$ resource utilization, disease burden, and the individual symptoms of abdominal pain, bloating, and changes in bowel habits. ${ }^{60}$ We previously demonstrated that overall IBS symptom severity is related to, but distinct from, HRQOL and is predicted by both GI and extraintestinal symptoms and disease-specific concerns. ${ }^{45}$ The differences in IBS symptom severity 
between pre- and postmenopausal women in our study may therefore not necessarily be explained by variations in one specific GI symptom, but rather be influenced by differences in psychological factors, cultural factors, perception of disease, and perceived physical HRQOL. The lack of significant individual symptom differences between groups could also be due at least in part to the fact that our IBS groups were heterogenous with respect to predominant bowel habit. Significant IBS bowel habit differences in the prevalence of individual symptoms ${ }^{61}$ and impact on daily activities have been reported. ${ }^{62}$

Interestingly, while we identified more severe overall IBS symptoms in postmenopausal women compared to premenopausal women, we did not find any significant differences in the severity of somatic symptoms between the two age groups of men and women with IBS. While preceding works evaluating somatic symptoms during the transition to menopause have overall shown mixed results, two previous studies demonstrated no difference in the frequency of somatic symptoms (eg, headaches, backaches) ${ }^{12,40}$ between pre- and postmenopausal IBS wome which collaborate the results of our study. The lack of differences in somatic symptoms may be due to the recruitment of IBS participants for physiologic studies, which exclude those with significant comorbidities.

The greater prevalence of constipation in women compared to men confirms prior studies. 6,8,9 Several animal and human models have demonstrated that female sex hormones can not only influence visceral sensitivity and visceromotor responses to painful stimuli, ${ }^{35,63,64}$ but also slow gastric emptying ${ }^{28-32}$ and intestinal transit. ${ }^{28,30,33,65}$ Estrogen and progesterone receptors are expressed throughout the GI tract, as well as conveyed through the smooth muscle cells of the GI tract and pelvic floor, afferent sensory fibers, and dorsal horn neurons in the spinal column. ${ }^{66-68}$ Although not statistically significant, we did see a numerically increased prevalence of IBS-C symptoms in both the postmenopausal women and agematched older men with IBS compared to their younger counterparts, which suggests that this particular finding may be an age-related phenomenon rather than specifically s relatedtothemenopausetransition. Thenon-significantincrease in proportion of postmenopausal women with IBS-C may in part be explained by a significant decrease in the percentage of loose or watery stools reported by postmenopausal compared to premenopausal women.

There were several limitations to our study. The methodology used to define menstrual phase did not allow for accurate reporting or recruitment of a sufficient number of women in the premenses or late luteal phases, periods when IBS symptoms are known to be heightened. More accurate determination of the menstrual cycle phase would have required measurements of the luteinizing hormone surge and serum progesterone levels to confirm ovulatory cycles. ${ }^{14}$ However, we previously found that progesterone levels correlated well with menstrual cycle phase based on LMP (unpublished results). We were not able to capture symptoms during premenses or menses (defined as days 1-3 of the menstrual cycle) with our questionnaire that assessed GI symptoms over the preceding week. Slightly over $20 \%$ of the premenopausal women with menstrual phase data were taking OCPs, although this was unlikely to affect our study results due to the lack of significant differences in OCP use between the different menstrual cycle phases. In addition, while a previous prospective study in premenopausal IBS women found that the use of OCPs was associated with less 
severe abdominal pain compared to women who were not taking OCPs, these differences were not significant after adjusting for multiple factors. ${ }^{14}$

There was also a relatively small number of postmenopausal IBS women and older IBS men in our study. In addition, the postmenopausal women included in our study ranged in age from 45-68 years, with a mean age of 54.8 years, making most of the women on the earlier side of postmenopause. It is therefore not known whether our results are completely generalizable to an older population of postmenopausal women. Additionally, there were more Asians in the younger groups, although we controlled for race in our analyses, so this was unlikely to affect our results. However, a larger study would n, be needed to better determine the relationship between race/ethnicity, menopause, and IBS.

In summary, our current study demonstrates that postmenopausal IBS women have significantly greater severity of overall IBS symptoms, as well as worse physical HRQOL compared to premenopausal women. However, these differences are not observed between age-matched older and younger IBS men. The modulatory effect of female sex hormones on brain-gut interactions which affect symptom severity likely contribute to these findings. Further research is needed to more fully determine the role that declining estrogenand progesterone levels during menopause, premenses, and menses exert on IBS symptoms.

\section{Supplementary Material}

Refer to Web version on PubMed Central for supplementary material.

\section{Acknowledgments}

Funding information

This study was funded via grants: P50 DK064539, R01 DK048351, R01 AT007137, and U54 123755

\section{REFERENCES}

1. Drossman DA. Functional gastrointestinal disorders: history, pathophysiology, clinical features, and Rome IV. Gastroenterology. 2016;150(6):1262-1279.e2.

2. Lovell RM, Ford AC. Global prevalence of and risk factors for irritable bowel syndrome: a metaanalysis. Clin Gastroenterol Hepatol. 2012;10:712-721.e4. [PubMed: 22426087]

3. Chial HJ, Camilleri M. Gender differences in irritable bowel syndrome. J Gend Specif Med. 2002;5:37-45. [PubMed: 12078061]

4. Lovell RM, Ford AC. Effect of gender on prevalence of irritable bowel syndrome in the community: systematic review and meta-analysis. Am J Gastroenterol. 2012;107:991-1000. [PubMed: 22613905]

5. Talley NJ. Diagnosing an irritable bowel: does sex matter? Gastroenterology. 1991;100:834-837. [PubMed: 1993508]

6. Chang L, Toner BB, Fukudo S, et al. Gender, age, society, culture, and the patients perspective in the functional gastrointestinal disorders. Gastroenterology. 2006;130:1435-1446. [PubMed: 16678557]

7. Simren M, Abrahamsson H, Svedlund J, Bjornsson ES. Quality of life in patients with irritable bowel syndrome seen in referral centers versus primary care: the impact of gender and predominant bowel pattern. Scand J Gastroenterol. 2001;36:545-552. [PubMed: 11346211]

8. Talley NJ, Boyce P, Jones M. Identification of distinct upper and lower gastrointestinal symptom groupings in an urban population. Gut. 1998;42:690-695. [PubMed: 9659166] 
9. Talley NJ, Zinsmeister AR, Melton LJ 3rd. Irritable bowel syndrome in a community: symptom subgroups, risk factors, and health care utilization. Am J Epidemiol. 1995;142:76-83. [PubMed: 7785677]

10. Berkley KJ. Sex differences in pain. Behav Brain Sci. 1997;20:371-380; discussion 435-513. [PubMed: 10097000]

11. Unruh AM. Gender variations in clinical pain experience. Pain. 1996;65:123-167. [PubMed: 8826503]

12. Lee OY, Mayer EA, Schmulson M, Chang L, Naliboff B. Gender-related differences in IBS symptoms. Am J Gastroenterol. 2001;96:2184-2193. [PubMed: 11467651]

13. Cohen H, Jotkowitz A, Buskila D, et al. Post-traumatic stress disorder and other co-morbidities in a sample population of patients with irritable bowel syndrome. Eur J Intern Med. 2006;17:567-571. [PubMed: 17142176]

14. Heitkemper MM, Cain KC, Jarrett ME, Burr RL, Hertig V, Bond EF. Symptoms across the menstrual cycle in women with irritable bowel syndrome. Am J Gastroenterol. 2003;98:420-430. [PubMed: 12591063]

15. Altman G, Cain KC, Motzer S, Jarrett M, Burr R, Heitkemper M. Increased symptoms in female IBS patients with dysmenorrhea and PMS. Gastroenterol Nurs. 2006;29:4-11. [PubMed: 16552294]

16. Heitkemper MM, Jarrett M, Cain KC, Shaver J, Walker E, Lewis L. Daily gastrointestinal symptoms in women with and without a diagnosis of IBS. Dig Dis Sci. 1995;40:1511-1519. [PubMed: 7628275]

17. Houghton LA, Lea R, Jackson N, Whorwell PJ. The menstrual cycle affects rectal sensitivity in patients with irritable bowel syndrome but not healthy volunteers. Gut. 2002;50:471-474. [PubMed: 11889064]

18. Drossman DA, Whitehead WE, Camilleri M. Irritable bowel syndrome: a technical review for practice guideline development. Gastroenterology. 1997;112:2120-2137. [PubMed: 9178709]

19. Schertzinger M, Wesson-Sides K, Parkitny L, Younger J. Daily fluctuations of progesterone and testosterone are associated with fibromyalgia pain severity. J Pain. 2018;19:410-417. [PubMed: 29248511]

20. Chai NC, Peterlin BL, Calhoun AH. Migraine and estrogen. Curr Opin Neurol. 2014;27:315-324. [PubMed: 24792340]

21. Lee JY, Choi HY, Ju BG, Yune TY. Estrogen alleviates neuropathic pain induced after spinal cord injury by inhibiting microglia and astrocyte activation. Biochim Biophys Acta Mol Basis Dis. 2018;1864:2472-2480. [PubMed: 29653184]

22. Tashiro A, Bereiter DA. The effects of estrogen on temporomandibular joint pain as influenced by trigeminal caudalis neurons. J Oral Sci. 2020;62:150-155. [PubMed: 32132330]

23. Sorge RE, Totsch SK. Sex differences in pain. J Neurosci Res. 2017;95:1271-1281. [PubMed: 27452349]

24. Wallace DM, Magnuson DJ, Gray TS. Organization of amygdaloid projections to brainstem dopaminergic, noradrenergic, and adrenergic cell groups in the rat. Brain Res Bull. 1992;28:447454. [PubMed: 1591601]

25. Seguela P, Watkins KC, Geffard M, Descarries L. Noradrenaline axon terminals in adult rat neocortex: an immunocytochemical analysis in serial thin sections. Neuroscience. 1990;35:249264. [PubMed: 2116602]

26. Tanaka M, Yoshida M, Emoto H, Ishii H. Noradrenaline systems in the hypothalamus, amygdala and locus coeruleus are involved in the provocation of anxiety: basic studies. Eur J Pharmacol. 2000;405:397-406. [PubMed: 11033344]

27. Heitkemper MM, Chang L. Do fluctuations in ovarian hormones affect gastrointestinal symptoms in women with irritable bowel syndrome? Gend Med. 2009;6(Suppl 2):152-167. [PubMed: 19406367]

28. Bond EF, Heitkemper MM, Perigo R. Gastric emptying and gastric-intestinal transit in rats with varying ovarian hormone status. Nurs Res. 1996;45:218-224. [PubMed: 8700655] 
29. Chen TS, Doong ML, Chang FY, Lee SD, Wang PS. Effects of sex steroid hormones on gastric emptying and gastrointestinal transit in rats. Am J Physiol. 1995;268:G171-G176. [PubMed: 7840200]

30. Wald A, Van Thiel DH, Hoechstetter L, et al. Gastrointestinal transit: the effect of the menstrual cycle. Gastroenterology. 1981;80:1497-1500. [PubMed: 7227774]

31. Hutson WR, Roehrkasse RL, Wald A. Influence of gender and menopause on gastric emptying and motility. Gastroenterology. 1989;96:11-17. [PubMed: 2909416]

32. Caballero-Plasencia AM, Valenzuela-Barranco M, Martin-Ruiz JL, Herrerias-Gutierrez JM, Esteban-Carretero JM. Are there changes in gastric emptying during the menstrual cycle? Scand J Gastroenterol. 1999;34:772-776. [PubMed: 10499477]

33. Bruce LA, Behsudi FM, Danhof IE. Smooth muscle mechanical responses in vitro to bethanechol after progesterone in male rat. Am J Physiol. 1978;235:E422-E428. [PubMed: 696863]

34. Emmanuel AV, Kamm MA. Laser Doppler flowmetry as a measure of extrinsic colonic innervation in functional bowel disease. Gut. 2000;46:212-217. [PubMed: 10644315]

35. Holdcroft A, Sapsed-Byrne S, Ma D, Hammal D, Forsling ML. Sex and oestrous cycle differences in visceromotor responses and vasopressin release in response to colonic distension in male and female rats anaesthetized with halothane. Br J Anaesth. 2000;85:907-910. [PubMed: 11732530]

36. Hinds JP, Stoney B, Wald A. Does gender or the menstrual cycle affect colonic transit? Am J Gastroenterol. 1989;84:123-126. [PubMed: 2916519]

37. Kamm MA, Farthing MJ, Lennard-Jones JE. Bowel function and transit rate during the menstrual cycle. Gut. 1989;30:605-608. [PubMed: 2731752]

38. Jackson NA, Houghton LA, Whorwell PJ, Currer B. Does the menstrual cycle affect anorectal physiology? Dig Dis Sci. 1994;39:2607-2611. [PubMed: 7995186]

39. Triadafilopoulos G, Finlayson M, Grellet C. Bowel dysfunction in postmenopausal women. Women Health. 1998;27:55-66. [PubMed: 9796084]

40. Cain KC, Jarrett ME, Burr RL, Rosen S, Hertig VL, Heitkemper MM. Gender differences in gastrointestinal, psychological, and somatic symptoms in irritable bowel syndrome. Dig Dis Sci. 2009;54:1542-1549. [PubMed: 18979200]

41. Choghakhori R, Abbasnezhad A, Amani R, Alipour M. Sex-related differences in clinical symptoms, quality of life, and biochemical factors in irritable bowel syndrome. Dig Dis Sci. 2017;62:1550-1560. [PubMed: 28374085]

42. Mearin F, Lacy BE, Chang L, et al. Bowel disorders. Gastroenterology. 2016;150:1393-1407.

43. Thompson WG, Longstreth GF, Drossman DA, Heaton KW, Irvine EJ, Muller-Lissner SA. Functional bowel disorders and functional abdominal pain. Gut. 1999;45(Suppl 2):ii43-ii47. [PubMed: 10457044]

44. Longstreth GF, Thompson WG, Chey WD, Houghton LA, Mearin F, Spiller RC. Functional bowel disorders. Gastroenterology. 2006;130:1480-1491. [PubMed: 16678561]

45. Spiegel B, Strickland A, Naliboff BD, Mayer EA, Chang L. Predictors of patient-assessed illness severity in irritable bowel syndrome. Am J Gastroenterol. 2008;103:2536-2543. [PubMed: 18637089]

46. Drossman DA. The functional gastrointestinal disorders and the Rome III process. Gastroenterology. 2006;130:1377-1390. [PubMed: 16678553]

47. Talley NJ, Phillips SF, Melton J 3rd, Wiltgen C, Zinsmeister AR. A patient questionnaire to identify bowel disease. Ann Intern Med. 1989;111:671-674. [PubMed: 2679285]

48. Kroenke K, Spitzer RL, Williams JB. The PHQ-15: validity of a new measure for evaluating the severity of somatic symptoms. Psychosom Med. 2002;64:258-266. [PubMed: 11914441]

49. Ware J Jr, Kosinski M, Keller SD. A 12-Item Short-Form Health Survey: construction of scales and preliminary tests of reliability and validity. Med Care. 1996;34:220-233. [PubMed: 8628042]

50. Ware JEKS, Kosinski M. SF-12: How to Score the SF-12 Physical and Mental Health Summary Scales. Boston, MA: Health Institute, New England Medical Center; 1995.

51. Zigmond AS, Snaith RP. The hospital anxiety and depression scale. Acta Psychiatr Scand. 1983;67:361-370. [PubMed: 6880820] 
52. Whitehead WE, Cheskin LJ, Heller BR, et al. Evidence for exacerbation of irritable bowel syndrome during menses. Gastroenterology. 1990;98:1485-1489. [PubMed: 2338190]

53. Jones BE, Yang TZ. The efferent projections from the reticular formation and the locus coeruleus studied by anterograde and retrograde axonal transport in the rat. J Comp Neurol. 1985;242:56-92. [PubMed: 2416786]

54. Bangasser DA, Eck SR, Ordones SE. Sex differences in stress reactivity in arousal and attention systems. Neuropsychopharmacology. 2019;44:129-139. [PubMed: 30022063]

55. Benarroch EE. Locus coeruleus. Cell Tissue Res. 2018;373:221-232. [PubMed: 28687925]

56. Berridge CW, Waterhouse BD. The locus coeruleus-noradrenergic system: modulation of behavioral state and state-dependent cognitive processes. Brain Res Brain Res Rev. 2003;42:3384. [PubMed: 12668290]

57. Bjelland I, Dahl AA, Haug TT, Neckelmann D. The validity of the Hospital Anxiety and Depression Scale. An updated literature review. J Psychosom Res. 2002;52:69-77. [PubMed: 11832252]

58. Lewis MJVHL, Whorwell PJ. Abdominal distension in females with irritable bowel syndrome: the effect of the menopause and hormone replacement therapy. Gut. 2001;48(Suppl. 1):168. [PubMed: $11156636]$

59. Han CJ, Dong C, Jarrett ME, Heitkemper MM. Symptom comparisons between Asian American and White American Women with irritable bowel syndrome. Gastroenterol Nurs. 2018;41:223232. [PubMed: 29847397]

60. Lembo A, Ameen VZ, Drossman DA. Irritable bowel syndrome: toward an understanding of severity. Clin Gastroenterol Hepatol. 2005;3:717-725. [PubMed: 16233998]

61. Schmulson M, Lee OY, Chang L, Naliboff B, Mayer EA. Symptom differences in moderate to severe IBS patients based on predominant bowel habit. Am J Gastroenterol. 1999;94:2929-2935. [PubMed: 10520847]

62. Ballou S, McMahon C, Lee HN, et al. Effects of irritable bowel syndrome on daily activities vary among subtypes based on results from the IBS in America Survey. Clin Gastroenterol Hepatol. 2019;17:2471-2478.e3. [PubMed: 31419572]

63. Giamberardino MA, Affaitati G, Valente R, Iezzi S, Vecchiet L. Changes in visceral pain reactivity as a function of estrous cycle in female rats with artificial ureteral calculosis. Brain Res. 1997;774:234-238. [PubMed: 9452216]

64. Ji Y, Murphy AZ, Traub RJ. Estrogen modulates the visceromotor reflex and responses of spinal dorsal horn neurons to colorectal stimulation in the rat. J Neurosci. 2003;23:3908-3915. [PubMed: 12736360]

65. Xiao ZL, Pricolo V, Biancani P, Behar J. Role of progesterone signaling in the regulation of Gprotein levels in female chronic constipation. Gastroenterology. 2005;128:667-675. [PubMed: 15765402]

66. Copas P, Bukovsky A, Asbury B, Elder RF, Caudle MR. Estrogen, progesterone, and androgen receptor expression in levator ani muscle and fascia. J Womens Health Gend Based Med. 2001;10:785-795. [PubMed: 11703891]

67. Waliszewski P, Blaszczyk M, Wolinska-Witort E, Drews M, Snochowski M, Hurst RE. Molecular study of sex steroid receptor gene expression in human colon and in colorectal carcinomas. J Surg Oncol. 1997;64:3-11. [PubMed: 9040793]

68. Campbell-Thompson M, Lynch IJ, Bhardwaj B. Expression of estrogen receptor (ER) subtypes and ERbeta isoforms in colon cancer. Cancer Res. 2001;61:632-640. [PubMed: 11212261] 


\section{Key Points}

- Menopause is associated with declining levels of estradiol and progesterone. Despite the well-known physiologic processes associated with menopause, little is known about the impact of the menopause transition on irritable bowel syndrome (IBS).

- This study determined that postmenopausal women with IBS have more severe IBS symptoms than premenopausal women, while interestingly, there were no comparable age-related changes seen in men with IBS.

- The modulatory effect of estradiol and progesterone on brain-gut interactions may contribute to these findings. 

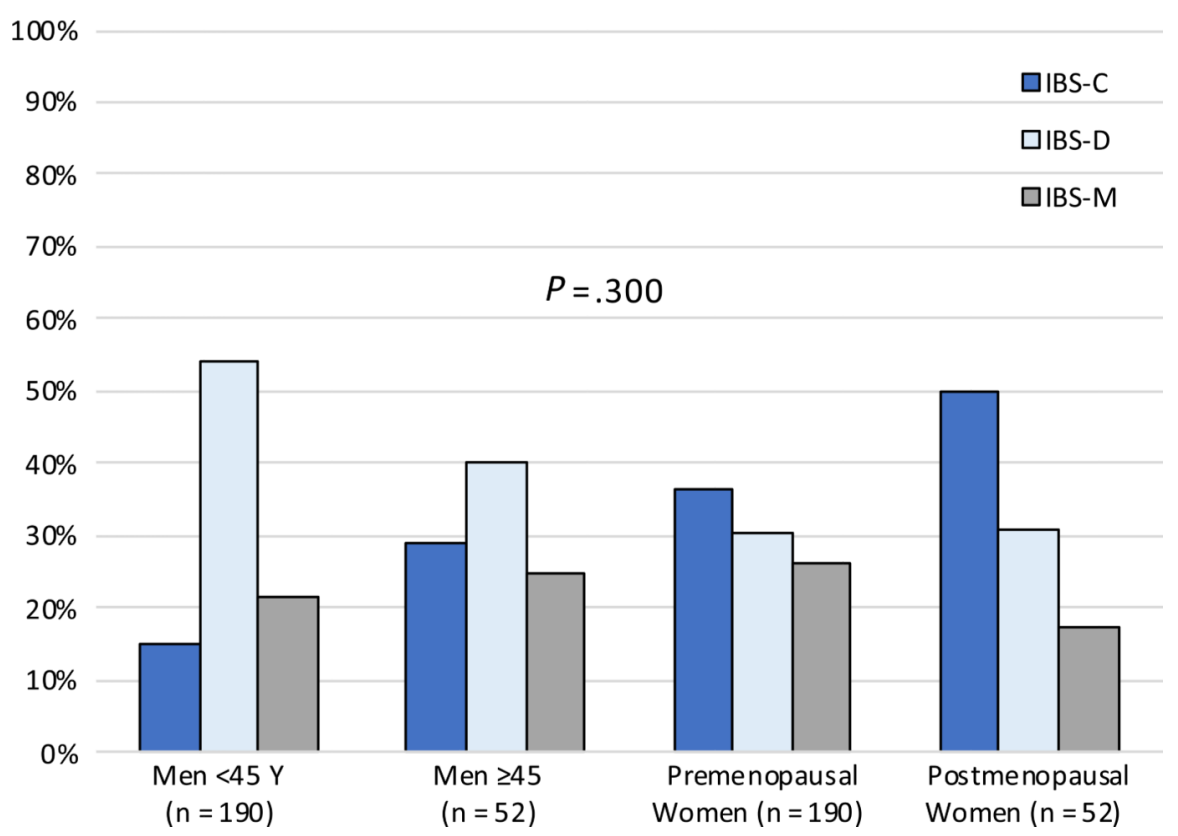

FIGURE 1.

Predominant irritable bowel syndrome (IBS) bowel habits in premenopausal women, postmenopausal women, younger men, and age-matched older men. Constipation increased with age for both men and women with IBS but was the predominant IBS subtype in women only. There was no significant relationship between age and gender for bowel habit subtype $(P=.300)$ 

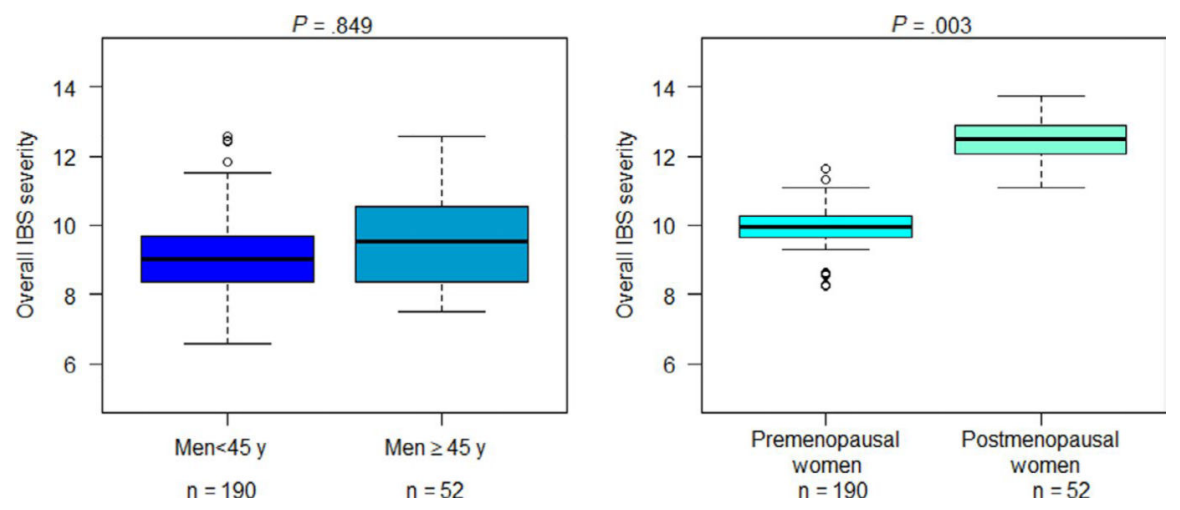

FIGURE 2.

Overall irritable bowel syndrome (IBS) symptom severity in premenopausal IBS women compared to postmenopausal IBS women and younger IBS men compared to age-matched older IBS men. Bowel Symptom Questionnaire (BSQ) overall IBS symptom severity score distributions among younger vs age-matched older IBS men and premenopausal vs postmenopausal IBS women. Box plots show median and interquartile ranges.

Postmenopausal IBS women had a greater severity of overall IBS symptoms compared to premenopausal women $(P=.003)$. No difference in severity of overall IBS symptoms in agematched older vs younger IBS men $(P>.05)$ 

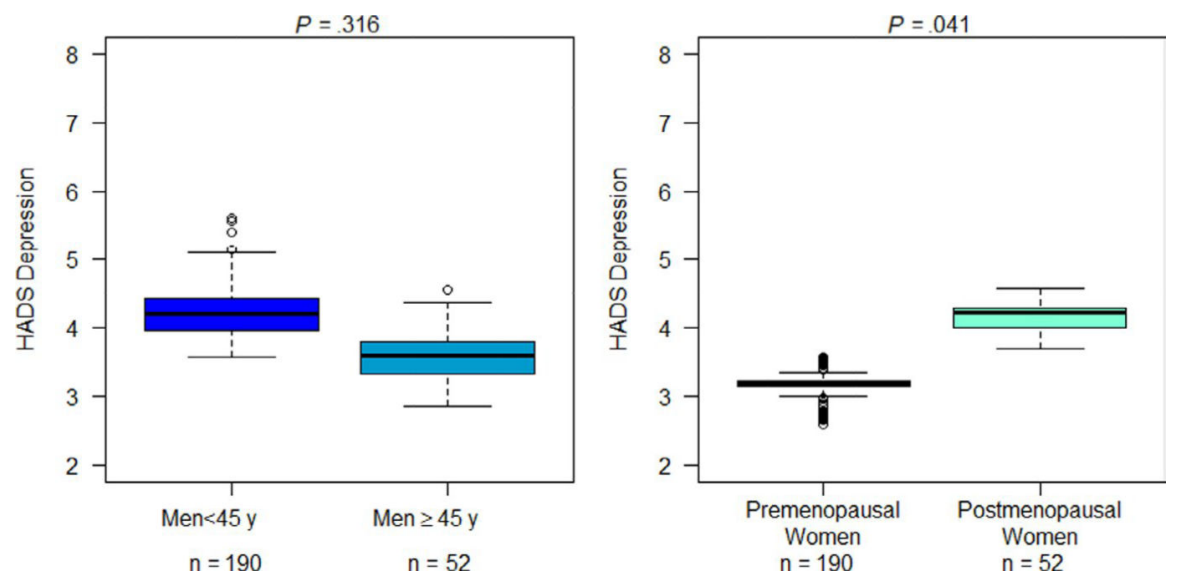

FIGURE 3.

Hospital Anxiety and Depression Scale (HADS) depression in premenopausal irritable bowel syndrome (IBS) women compared to postmenopausal IBS women and younger IBS men compared to age-matched older IBS men. Hospital Anxiety and Depression Scale depression score distributions among younger vs age-matched older IBS men and premenopausal vs postmenopausal IBS women. Box plots show median and interquartile ranges. Postmenopausal IBS women had higher depression symptom scores than premenopausal IBS women $(P=.041)$, whereas depression scores were similar between agematched older and younger IBS men $(P=.316)$ 

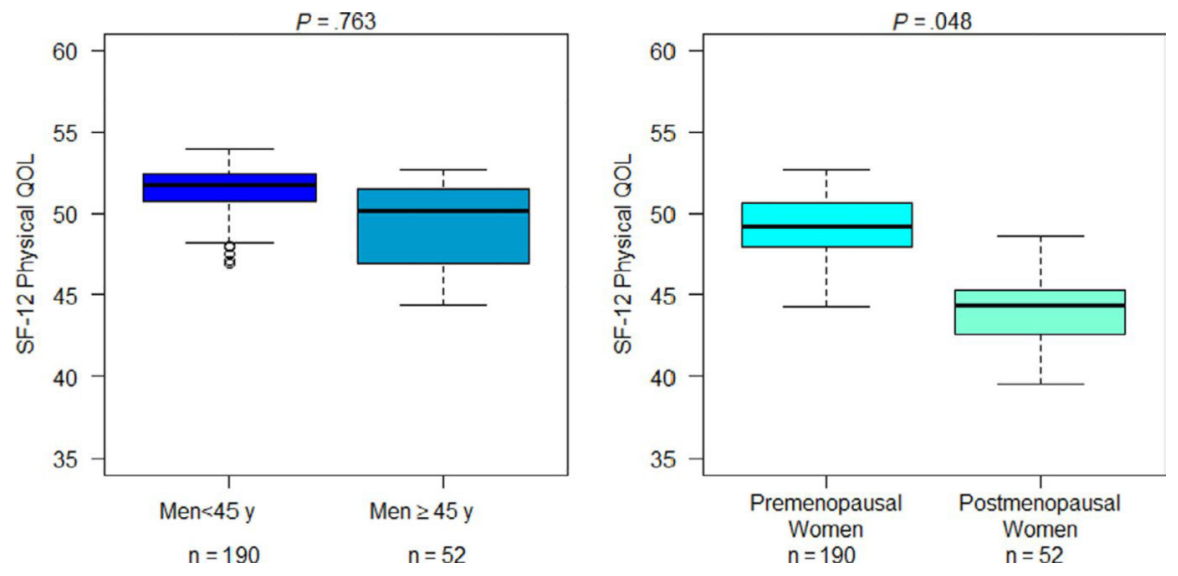

FIGURE 4.

Physical quality of life in premenopausal irritable bowel syndrome (IBS) women compared to postmenopausal IBS women and younger IBS men compared to age-matched older IBS men. Short Form (SF-12) Physical Composite Score distributions among younger vs older IBS men and premenopausal vs postmenopausal IBS women. Box plots show median and interquartile ranges. Postmenopausal IBS women had lower physical health-related quality of life (HRQOL) scores compared to premenopausal IBS women $(P=.048)$. There was no significant difference in physical HRQOL scores for age-matched older compared to younger IBS men 


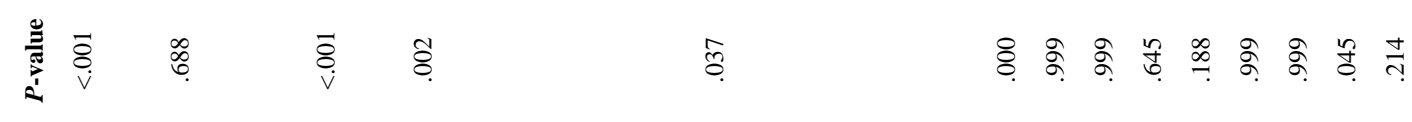

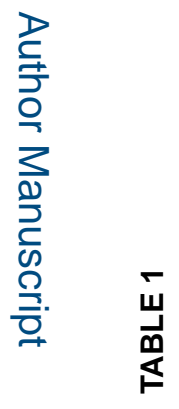

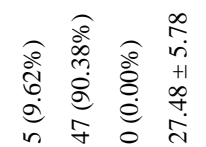

$\stackrel{8}{\circ}$

菅. 


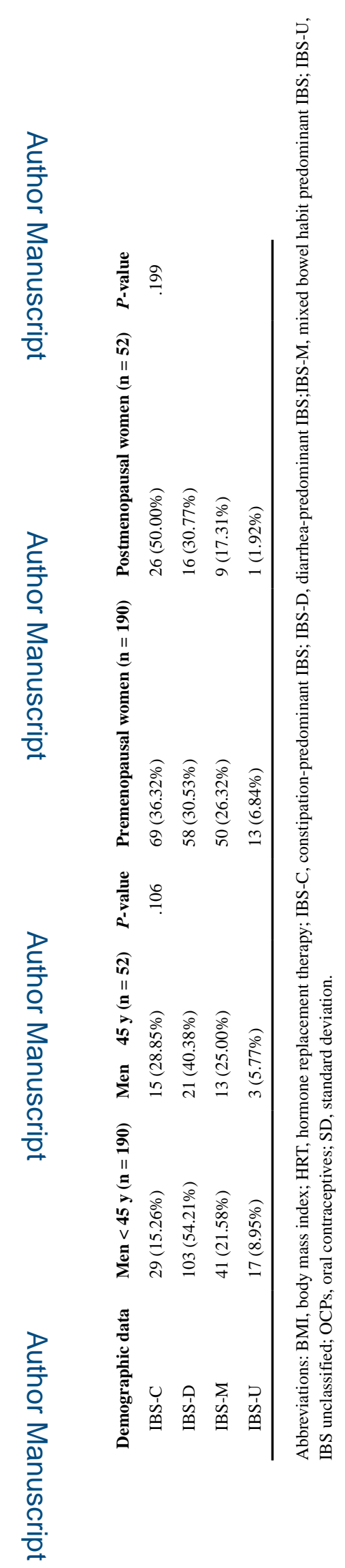

Neurogastroenterol Motil. Author manuscript; available in PMC 2021 October 01. 


\section{TABLE 2}

Comparison of characteristics between premenopausal women and postmenopausal women with IBS and men with IBS incomparable age categories

\begin{tabular}{|c|c|c|c|c|c|c|}
\hline & $\begin{array}{l}\text { Men }<45 \text { y }(\mathrm{n} \\
=190)\end{array}$ & $\begin{array}{l}\text { Men } \geq 45 \text { y }(n \\
=52)\end{array}$ & $P$-value ${ }^{\dagger}$ & $\begin{array}{l}\text { Premenopausal women } \\
(n=190)\end{array}$ & $\begin{array}{l}\text { Postmenopausalwomen }(n= \\
\text { 52) }\end{array}$ & $P$-value ${ }^{\dagger}$ \\
\hline $\begin{array}{l}\text { Overall IBS } \\
\text { symptom } \\
\text { severity }\end{array}$ & $9.51 \pm 4.57$ & $9.48 \pm 4.33$ & .849 & $9.98 \pm 4.27$ & $12.39 \pm 4.51$ & $.003^{*}$ \\
\hline Abdominal pain & $8.34 \pm 4.57$ & $8.27 \pm 4.73$ & .866 & $9.48 \pm 4.52$ & $10.56 \pm 4.45$ & .422 \\
\hline Bloating & $9.36 \pm 5.47$ & $8.71 \pm 4.98$ & .953 & $11.36 \pm 5.12$ & $12.38 \pm 5.32$ & .451 \\
\hline PHQ-15 & $9.28 \pm 4.12$ & $9.19 \pm 5.30$ & .212 & $11.82 \pm 4.83$ & $12.27 \pm 5.20$ & .991 \\
\hline $\begin{array}{l}\text { HAD } \\
\text { depression }\end{array}$ & $4.44 \pm 3.81$ & $4.55 \pm 3.13$ & .316 & $3.27 \pm 2.87$ & $4.20 \pm 3.28$ & $.041^{*}$ \\
\hline HAD anxiety & $8.04 \pm 4.59$ & $7.05 \pm 4.18$ & .247 & $7.62 \pm 4.31$ & $7.59 \pm 5.21$ & .600 \\
\hline SF-12 PCS & $49.91 \pm 8.31$ & $46.57 \pm 9.52$ & .763 & $49.04 \pm 9.18$ & $43.62 \pm 9.42$ & $.048^{*}$ \\
\hline SF-12 MCS & $44.90 \pm 10.30$ & $45.83 \pm 9.88$ & .516 & $45.61 \pm 9.73$ & $45.89 \pm 11.02$ & .304 \\
\hline
\end{tabular}

Abbreviations: HAD, Hospital Anxiety and Depression; IBS, irritable bowel syndrome; PHQ-15, Patient Health Questionnaire; SF-12 MCS, SF-12 Mental Composite Score; SF-12 PCS, SF-12 Physical Composite Score.

${ }^{\dagger}$ P-values comparing younger men to age-matched older men.

${ }^{t}$ P-values comparing premenopausal to postmenopausal women. 


\section{TABLE 3}

Menstrual cycle variations in IBS symptoms, somatic symptoms, psychologic symptoms, and HRQOL

Overall IBS symptom severity
Abdominal pain
Bloating
PHQ-15
HAD depression
HAD anxiety
SF12 PCS
SF12 MCS

$\begin{array}{rrl}\text { Follicular }(\mathbf{n}=\mathbf{3 6}) & \text { Luteal }(\mathbf{n = 7 4}) & \boldsymbol{P} \text {-value } \\ 9.64 \pm 3.99 & 10.16 \pm 4.21 & .39 \\ 9.54 \pm 4.39 & 9.46 \pm 4.40 & .935 \\ 11.14 \pm 4.83 & 11.85 \pm 5.42 & .373 \\ 11.55 \pm 4.28 & 12.37 \pm 5.40 & .534 \\ 2.53 \pm 2.08 & 3.84 \pm 3.33 & .073 \\ 6.86 \pm 3.77 & 7.96 \pm 4.60 & .338 \\ 49.33 \pm 8.51 & 50.16 \pm 7.42 & .780 \\ 47.14 \pm 9.20 & 44.68 \pm 9.39 & .243\end{array}$

Abbreviations: HAD, Hospital Anxiety and Depression; HRQOL, health-related quality of life; IBS, irritable bowel syndrome; PHQ-15, Patient Health Questionnaire; SF-12 MCS, SF-12 Mental Composite Score;SF-12 PCS, SF-12 Physical Composite Score. 